\title{
Quantification of the Effects of Small Mistilts on Dopant Visibility in Nanocrystals.
}

Jacob T. Held ${ }^{1}$, Samuel Duncan ${ }^{1}$, and K. Andre Mkhoyan ${ }^{1}$

1. Department of Chemical Engineering \& Materials Science, University of Minnesota, Minneapolis, MN 55455.

Aberration-corrected annular dark field scanning transmission electron microscopy (ADFSTEM) has been demonstrated to be a powerful technique for the visualization of individual dopant atoms. ${ }^{1}$ Provided the dopant has a higher atomic number than the host, dopant atoms can be clearly visible in ADF-STEM images of thin, lightly doped specimens and can be readily located to an atomic site in two dimensions given adequate lateral resolution. Channeling of the electron probe down atomic columns in aligned crystalline samples causes the visibility of dopant atoms to change predictably, providing a useful method of determining the dopant atom location also in third dimension. ${ }^{2}$

As discussed by Xin, et al. ${ }^{3}$, tilting away from a high order zone axis mitigates channeling effects in crystalline specimens and allows for more readily interpretable through-focal series. This and quantitative STEM imaging of doped crystals aligned to high-order zone axes ${ }^{4}$, have been shown to be powerful techniques for analyzing film specimens. However, nanocrystal specimens present a unique array of challenges. Two of these challenges are obtaining an accurate measurement of crystal orientation, and controlling beam-induced rotation of the nanocrystal. The goal of this study is to evaluate the effect of small inaccuracies in crystal alignment on the visibility of dopant atoms in nanocrystals.

We employed multislice simulations of ADF-STEM imaging of $2.5 \mathrm{~nm}$ Ge-doped Si nanocrystals to evaluate these effects. The TEMSIM package ${ }^{5}$ was used to simulate aberrationcorrected ADF-STEM images with accelerating voltage $100 \mathrm{keV}$, convergence semi-angle 25 $\mathrm{mrad}, \mathrm{C}_{\mathrm{s}(3)}=-0.015 \mathrm{~mm}, \mathrm{C}_{\mathrm{s}(5)}=10 \mathrm{~mm}, \mathrm{df}=-30 \AA$, and 54/200 inner/outer detector collection angles. Thermal vibration was included by averaging 10 phonon configurations at $300 \mathrm{~K}$. The spherical $2.5 \mathrm{~nm}$ nanocrystals are tilted within the TEMSIM code to $0,2,10$, and 24 mrad in two orthogonal directions off of the [001], [110], and [111] zone axes. Images were generated with Ge dopants placed at each vertical lattice position in the central column of the nanocrystal. Example images are shown in Figure 1. By comparing the dopant visibility in tilted images, we found that slight differences in mistilt contributed significantly to the uncertainty in vertical dopant location, as shown in Figure 2. This means that precision in determining the orientation of the specimen is a key factor in analyzing the 3D location of individual dopant atoms in ADF-STEM imaging. ${ }^{6}$

References:

[1] V. C. Holmberg, et al. Chem. Mater. 25 (2013) p. 1332.

[2] A. Mittal, et al. Ultramicroscopy 111 (2011) p. 1101.

[3] H. L. Xin, et al. Appl. Phys. Lett. 92 (2008) p. 013125. 
[4] J. Hwang, et al. Phys. Rev. Lett. 111 (2013) p. 266101.

[5] E.J. Kirkland, Advanced Computing in Electron Microscopy, (Springer, New York, 2010).

[6] This work was supported primarily through the NSF under Award No. DMR-1410888 and in part by NSF MRSEC under Award No. DMR-1420013.
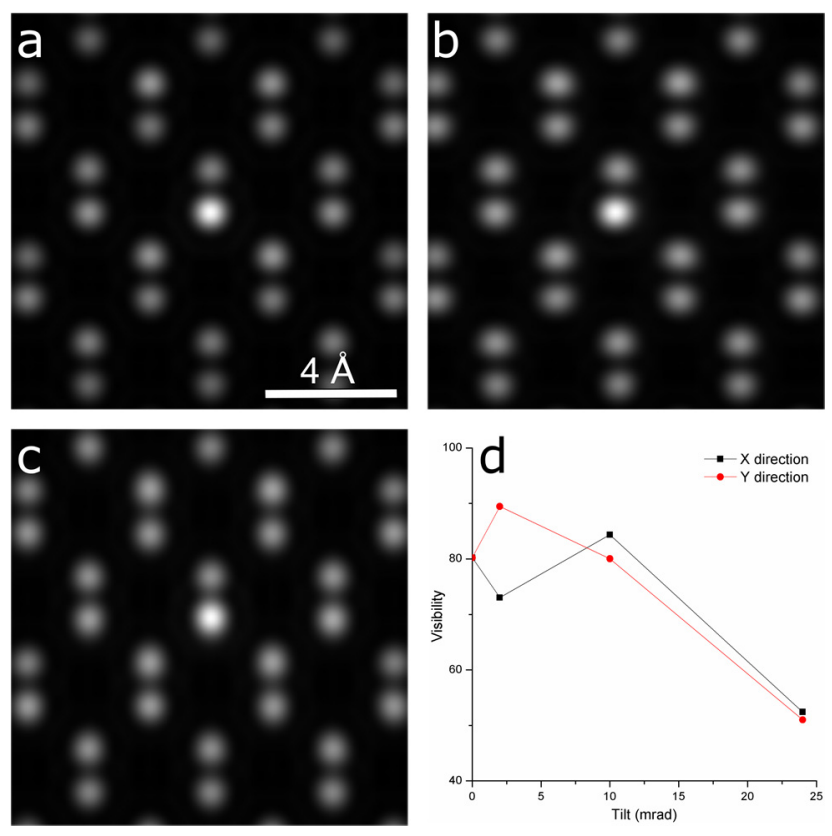

Figure 1. (a) Simulated ADF-STEM image of a $2.5 \mathrm{~nm}$ Si nanocrystal aligned along the [110] zone axis with a Ge atom substituted in the sixth lattice site from the entrance surface in the middle column of the nanocrystal. (b) and (c) show the same crystal, tilted $24 \mathrm{mrad}$ in the y-direction and the $\mathrm{x}$-direction, respectively. (d) Dependence of visibility on tilt for this dopant in the $\mathrm{x}$ - and $\mathrm{y}$ directions.
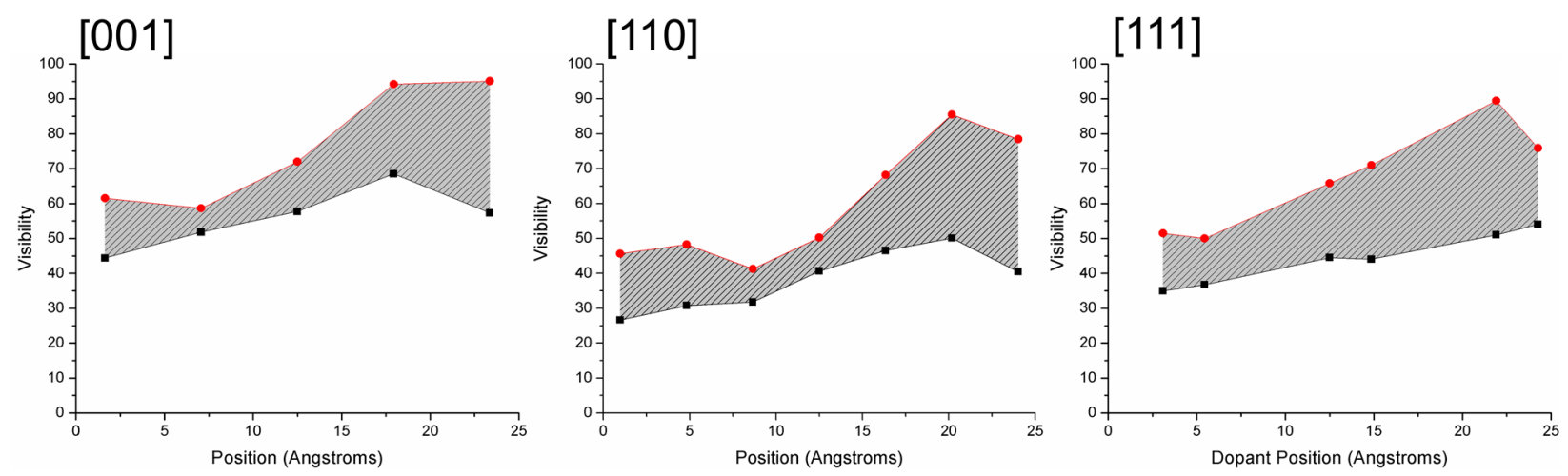

Figure 2. Minimum and maximum visibilities due to mistilt (0, 2, 10, and $24 \mathrm{mrad})$ for dopants at each vertical lattice position near the [001], [110], and [111] zone axes. 